\title{
While My Guitar Gently Weeps. Zur SOUND-, Groove- Und PERformance- VIRTUOSITÄT IN DER POPULÄREN MUSIK
}

\author{
Dirk Stederoth
}

Zuletzt 2012 tourten die »G3«, also Steve Morse, Joe Satriani und Steve Vai, den Zappa seinen »Stunt-Gitarristen « nannte, durch die Lande und zeigten aller Welt, was Virtuosität auf der Rock/Pop-Gitarre zu bedeuten hat. In Fortsetzung der Tradition, die bereits in den 1980er Jahren mit John McLaughlin, Al Di Meola und Paco de Lucía und der Nacht in San Francisco ihren Anfang nahm, dudeln die G3 schon seit 1996 in unterschiedlichen Besetzungen und atemberaubender Fingergeschwindigkeit über die Bretter, um zu zeigen, was s state of the art des Gitarrenspiels ist. Hierbei folgen sie einem Virtuosenbegriff, der, wenn nicht im Piano-Battle von Franz Liszt und Sigismund Thalberg in den 1830er Jahren seinen Hintergrund, so doch zumindest in Paganini seinen Ahnherrn hat. Dies wird jedenfalls sehr deutlich in Interviews, die Satriani und Vai im September 2016 für die Zeitschrift Gitarre \& Bass gegeben haben. Satriani, auf Virtuosität in der Rockmusik hin angesprochen, antwortet hier selbstkritisch, er sei »der am wenigsten technisch versierte «, zudem gibt er kund, »dass ich der Langsamste in diesem Haufen bin « und er »immer noch mit dem Gefühl zu kämpfen hat, dass die Gitarre schwierig zu spielen ist «, weshalb er »beim Spielen vielleicht eine Erfolgsrate von 85\% [habe], während die anderen Jungs fast jeden Abend die 100\% erreichen « (Menge 2016: 33). Diese klare Bestimmung des Virtuosenbegriffs ergänzt sich sehr schön durch die Bestimmung von Vai: »Mein Ziel war es immer, herausragende technische Fähigkeiten auf dem Instrument zu erlangen, ich mag das einfach und dafür konnte ich mich begeistern. Es fühlt sich toll an, dieses Instrument fast mühelos spielen zu können« (ebd.: 39). Die technische Versiertheit auf einem Instrument, die Fähigkeit, komplexe tonale Gebilde in rasender Schnelligkeit spielen zu können, scheinen also immer noch die bestimmenden Merkmale für den Virtuosenbegriff zu sein, was wie gesagt bereits für das 19. Jahrhundert galt. 
Dieser Virtuosenbegriff wurde aber schon im 18. Jahrhundert eher negativ besetzt (vgl. Reimer 1973) und ihm die positive Form eines Virtuosen im Ausdruck gegenübergestellt. Auch im 19. Jahrhundert wurde Virtuosität dann nicht nur gefeiert, wenn etwa Hugo Riemann in den 1890er Jahren anprangert, man sei

»heute längst dabei angekommen, den Virtuosen um seiner Virtuosität willen anzustaunen, gleichviel auf welche Weise er dieselbe bethätigt, so dass das inhaltsloseste Phrasenwerk, der jämmerlichste Firlefanz und musikalische Hokuspokus das Konzertpublikum zu >frenetischem Applaus< hinzureissen vermag« (Riemann 1967: 3).

Vor diesem Hintergrund stellt sich die Frage, ob der Virtuosenbegriff nicht auch anders zu bestimmen wäre, zumal wenn man sich die Frage vorsetzt, was denn virtuositas, also Tugend im weiteren Sinne in der Musik zu bedeuten hat.

Wenn Riemann davon spricht, dass solche Virtuosenpräsentationen häufig das »inhaltsloseste Phrasenwerk « zum Besten geben, dann richtet sich das wohl darauf, dass es in ihnen nicht um eine besondere komponierte Struktur und ihre angemessene Interpretation geht, als vielmehr darum, in variativen Phrasen ihre technischen Fähigkeiten zur Schau zu stellen. Gleiches kann für Fälle wie die G3 im Rahmen der populären Musik gelten, insofern solche virtuosen Präsentationen von Popmusik-Klassikern in gleicher Weise wenig songorientiert sind und ebenso die Darstellung ihrer technischen Fertigkeiten als vordergründiges Ziel intendieren.

Es fragt sich also, ob nicht jenseits der Zurschaustellung technischer Fähigkeiten sinnvoll von Virtuosität gesprochen werden kann. Dies sei im Folgenden in zwei Schritten untersucht, wobei zunächst nach einer anderen Bestimmung von Virtuosität im Rahmen strukturorientierter Musik gefragt wird, um von dieser ausgehend dann eine eigenständige Bestimmung dieses Begriffs im Rahmen der populären Musik vorzunehmen.

\section{Die tugendhafte Interpretation}

Wenn hier von strukturorientierter Musik die Rede ist, so ist damit gemeint, dass es bei ihr um die Interpretation einer festgelegten Struktur bzw. eines Werkes zu tun ist, das in Form einer Partitur oder Ähnlichem vorliegt. Dass dabei der Grad der Festlegungen und Freiheiten innerhalb dieser Struktur unterschiedlich hoch sein kann, spielt zunächst keine Rolle - wichtig ist hier lediglich, dass die in einer Partitur oder Ähnlichem niedergelegte musi- 
kalische Struktur all das enthält, was dem Komponisten als zentral für die musikalische Idee erschien, die er in dieser Struktur auszudrücken sich vorgenommen hat. Eine Interpretation hat sich entsprechend an dieser musikalischen Idee, wie sie in der Partitur niedergelegt ist, zu orientieren, wobei ihr so viele Freiräume bleiben, wie sie von jener zugelassen sind. Paradigmatisch für eine solche strukturorientierte Musik, insofern sie einen sehr hohen Grad an Festlegungen beinhaltet, ist beispielsweise die klassische Kunstmusik etwa von der Wiener Klassik bis zur Zwölftonmusik von Schönberg und Webern. Aber Zappa ist das beste Beispiel dafür, dass solche strukturorientierte Musik auch in anderen Genres vorkommen kann, war für ihn in seiner späten Phase u.a. das Vermeiden von Fehlern und Freiheiten der Grund dafür, lieber mit dem Synclavier zu arbeiten als mit einer Band. ${ }^{1}$

Doch zum Thema: Was ist wirklich virtuos, also tugendhaft bei der Interpretation einer Partitur? Ich möchte dies in drei Bereichen thematisieren: einmal im Bereich der Interpretation des Tonalen, die sich in der Instrumentierung und Klangfarbe kundgibt; zweitens in der Interpretation der Rhythmik und Metrik, die sich an der Phrasierung und agogischen Dynamik zeigt; und schließlich bezogen auf die gesamte Komposition, die sich in der Gesamtinterpretation als Einheit der beiden vorausgehenden Momente darstellt sowie im Zusammenspiel der einzelnen Instrumente sich ausdrückt.

Um mit der Tonalität zu beginnen, so möchte ich für diese Thematisierung auf ein Zitat aus Adornos dritter Vorlesung über die »Funktion der Farbe in der Musik« zurückgreifen, in der er sagt:

»[E]s wäre nämlich an der Zeit, daß die Instrumentalisten selber sich dessen bewußt werden, daß die strukturelle Darstellung von Musik in weitem Maße nur möglich ist durch die bewußte Verfügung über die Timbres, die dem einzelnen Instrument und die der Singstimme zur Verfügung stehen [...] und diese Seite der instrumentalen Wiedergabe, nämlich daß jedes Instrument innerhalb seiner Möglichkeiten sich soweit schattieren muß, soweit verschiedene Klangfarben benutzen muß, wie sie in der Komposition [...] angelegt sind, das ist eigentlich einer der Punkte, von denen man sagen kann, daß nach wie vor die Technik der instrumentalen Wiedergabe hinter den Anforderungen der Kompositionen selber vollständig zurückgeblieben sei« (Adorno 2014: 527).

1 »Substracting the bullshit and the mistakes [played by Live-Musicians, D.S.], if I had to choose between live musicians or La Machine, I must admit, from time to time I'm almost tempted to opt for the shuman element « (Zappa 1989: 173). - Ob das eine gute Idee ist, fragt sich angesichts seiner LP Jazz From Hell (1986), die bis auf einen Song vom Synclavier eingespielt wurde. 
Worauf Adorno hier verweist, ist der Anspruch, dass eine tonale Interpretation nicht nur die einfache Wiedergabe der in der Partitur notierten Töne und Harmonien ist, sondern dass sich die Intonation und farbliche Gestaltung der einzelnen Töne von der Idee der Komposition her bestimmen muss oder besser: sollte. Idealiter hat jeder Ton aufgrund seiner spezifischen Funktion im gesamten Kompositionsgefüge eine eigene Färbung, die der Interpret angesichts der Idee der Komposition auszuführen bzw. einzustimmen hat. Eine solche Konzentration auf die jeweilige Färbung einzelner Töne in ihrer Bezogenheit auf das Ganze der Komposition hin, wäre in diesem Sinne die höchste Form von tonaler Virtuosität im Rahmen einer strukturorientierten bzw. notationsbezogenen Musik.

Entsprechend verhält es sich dann auch im Bereich der Rhythmik und Metrik, insofern auch hier die bloße 1:1-Übertragung der rhythmischen Strukturen der Partitur für eine angemessene, oder eben virtuose Interpretation nicht ausreicht. Riemann hatte entsprechend im ausgehenden 19. Jahrhundert durch die Entwicklung eines ergänzenden Zeichensystems versucht, dieser Problematik gerecht zu werden, insofern seine agogischdynamischen Zeichen dem Komponisten die Möglichkeit geben sollten, die Lebendigkeit im interpretativen Ausdruck notierbar zu machen (vgl. Riemann 1884). Im Hintergrund dieses Bestrebens liegt natürlich, dass eine notierte Melodie erst durch die spezifische Phrasierung an Lebendigkeit gewinnt, wobei man auch hier davon ausgehen muss, dass diese Lebendigkeit von der Idee des Werkes her bestimmt sein sollte und eben nicht von der bloßen Individualität des Interpreten. Rhythmische Virtuosität im Rahmen einer Interpretation zeichnet sich demnach dadurch aus, dass die Melodien und rhythmischen Gebilde einer Partitur von der Idee des Werkes her in ihrer jeweiligen Eigenheit phrasiert werden müssen, so dass jedes einzelne rhythmische Gebilde in seiner kompositorischen Funktion für die ganze Komposition adäquat interpretiert wird.

Blickt man nun drittens auf die Komposition als Ganzes so integrieren sich einmal die beiden geschilderten Momente zu einer Einheit und zudem kommt noch das Zusammenspiel der einzelnen Instrumente als ein weiteres Moment in den Blick. Diesbezüglich möchte ich auf eine Passage aus Adornos Einleitung in die Musiksoziologie verweisen, in der er sich mit dem adäquaten Spiel in einem Kammerorchester beschäftigt. Sie lautet:

»Der erste Schritt, Kammermusik richtig zu spielen, ist, zu lernen, nicht sich aufzuspielen, sondern zurückzutreten. Das Ganze konstituiert sich nicht durch die auftrumpfende Selbstbehauptung der einzelnen Stimmen - sie ergäbe ein barbarisches Chaos - sondern durch einschränkende Selbstreflexion. [...] Große Kammermusikspieler, die im Geheimnis der Gattung sind, 
neigen dazu, so sehr auf den anderen zu hören, daß sie den eigenen Part nur markieren« (Adorno 1975: 109f.).

Dieses Zitat zeigt sehr schön, dass ein virtuoses Zusammenspiel nur durch ein adäquates Einpassen der eigenen Stimme in den Gesamtzusammenhang möglich ist, insofern auch das Zusammenspiel nicht der Demonstration individueller Fähigkeiten zu dienen hat, sondern vielmehr der angemessenen Darstellung der Idee der Komposition. Hier wird der Unterschied zu dem landläufigen Virtuosenbegriff wohl am deutlichsten, insofern es diesem ja wie gesagt primär um die Darstellung von individuellen technischen Fähigkeiten geht und eben nicht um die Orientierung an der überindividuellen Idee der Komposition, wie sie für die hier vorgeschlagenen Virtuositätsbegriffe, also tonale, rhythmische und Ensemble-Virtuosität, wie man es vielleicht nennen könnte, einschlägig sind. Die Partitur bzw. die Idee des Werks hat hier das letzte Wort und virtuos kann in dieser Hinsicht nur derjenige sein, der dieses Wort am adäquatesten artikulieren kann.

\section{Sound-, Groove- und Performance-Virtuosität}

In einem zweiten Schritt sei nun die Frage untersucht, ob für Musik, die nicht primär von einer musikalischen Struktur, sondern vielmehr von der musikalischen Realisierung ausgeht, was für die meiste populäre Musik gelten kann, vergleichbare Virtuositätsformen bestimmbar sind. Zur Einstimmung auf diese Frage, sei zunächst noch einmal auf das erwähnte Interview mit Satriani zurückgekommen. Mit Bezug zu seiner Einschätzung, dass er sich nicht für einen virtuosen Gitarristen hält, kommt er dann auch auf die Beatles zu sprechen:

»Schau dir die Beatles an. Keiner von ihnen war im klassischen Sinne virtuos. Da hört man nie Zeug, das sehr schnell oder kompliziert wäre. Aber sie haben tolle Musik gemacht, großartige Songs geschrieben, sehr gut zusammen gespielt und gesungen. [...] Die meisten Bands, die ich mochte, waren so, und wenn, wie zum Beispiel bei Led Zeppelin, auch mal hervorragende Musiker spielten, dann stellten sie das nicht in den Vordergrund. Die eigene Karriere oder dein Können haben nichts in einem Song verloren« (Menge 2016: 34).

Und entsprechend fällt dann auch die Auswahl seiner Gitarristen-Vorbilder aus: »Mich haben immer Gitarristen fasziniert, die sich auf den Song konzentriert haben. Die Vorbilder für mein Spiel waren George Harrison, Keith Richards, Jimi Hendrix und Jimmy Page« (ebd.). 
Bezieht man diese Aussagen von Satriani auf das, was oben über die Virtuosität in der strukturorientierten Musik ausgeführt wurde, dann könnte man einmal sagen, dass er zwar immer noch dem, wie er es nennt, »klassischen Virtuosenbegriff « anhängt, jedoch eigentlich ganz ähnliche Tugenden von Rock-Gitarristen beschreibt, wie sie oben in Abgrenzung zu jenem Begriff von Virtuosität entfaltet wurden. Am Song orientiert sein, gutes Zusammenspiel, ohne sich in den Vordergrund zu rücken, das wären die eigentlichen virtuositas der Rockinstrumentalisten, und eben nicht technisches Artistentum. So verweist dann Satriani in Bezug auf das Spiel von Rocksoli auf u.a. George Harrison, »bei dem auch jedes Solo speziell an den Song angepasst war. Das war nie: >Ich bin George Harrison und so klingt es, wenn ich ein Solo spiele.< Eine Einstellung, die ja in den 80ern populär wurde. Da war jedes Solo eine Zirkusnummer « (ebd.: 34f.). Kurz: wenn man den oben entfalteten Virtuosenbegriffen folgt, sind es gerade nicht die Zirkusnummern, durch die sich wirkliche Virtuosen auch in der Rockmusik auszeichnen, sondern vielmehr die songorientierte Anpassung des eigenen Spiels.

Nimmt man vor diesem Hintergrund einen Song wie beispielsweise »While My Guitar Gently Weeps«, so wären etwa die beiden sehr songdienlichen Soli von Eric Clapton aus der Beatles-Originalversion als wesentlich virtuoser zu bezeichnen, als das sehr artistisch gestaltete Cover von Jeff Healey aus den späten 1980ern, das fraglos technisch herausragend gespielt ist, jedoch das Solo und nicht den Song in den Vordergrund stellt und das zumal in einer für Jeff Healey typischen Weise.

Jedoch drängt sich hier sofort eine nicht ganz unerhebliche Frage auf: Was ist denn eigentlich der Song »While My Guitar Gently Weeps « und was ist seine Idee? Wollte man die oben erläuterten Virtuosenbegriffe auf die populäre Musik übertragen, müsste es auch eine entsprechende Idee eines Songs geben, an der sich das Spiel entsprechend anmessen kann. Doch was wäre in diesem Fall eine solche Idee? Harrison schreibt über die Entstehung dieses Songs, dass er, inspiriert durch das I Ging, eine Zufallsentscheidung treffen wollte und eine beliebige Passage aus einem Buch zum Thema erheben wollte - dieses zufällig gefundene Thema war dann »Gently Weeps «. ${ }^{2}$

2 Vgl. Harrison 1980: 120: »Around the time of writing While My Guitar Gently Weeps I had a copy of the >I Ching - the (Chinese) Book of Changes which seemed to me to be based on the Eastern concept that everything is relative to everything else, as opposed to the Western view that things are merely coincidental. This idea was in my head when I visited my parents' house in the North of England. I decided to write a song based on the first thing I saw upon opening any book - as it would be relative to that moment at that time. I picked up 
Doch ist dies nun die Idee des Songs? Für die Originalversion scheint die »sanft weinende Gitarre in der Tat im Hintergrund der Soundgestaltung die Feder geführt zu haben, denn diese Sound-Verbindung von Chorus, Crunch und Tremolo, die den E-Gitarrensound bestimmt, hat in der Tat etwas Wimmerndes. Und auch die Spielart der Sologitarre nutzt die vielen Bendings, um einen klagenden Sound zu generieren. Dann ist auch die kaum vernehmbare Orgel lediglich dafür eingesetzt, dem Gitarrensound etwas sphärischkreischendes zu geben. Und schließlich gibt neben den Dur-Moll-Wechseln in der harmonischen Struktur auch der Groove des Songs, betont durch das Schluchzen der sich schließenden HiHat am Anfang des Songs, noch die angemessene harmonische und rhythmische Basis. Kurz: Hier wird in der Tat alles getan, um der »sanft weinenden Gitarre « einen Ton zu verleihen. Und keine Coverversion hat es bisher in vergleichbarer Weise geschafft, auch nicht die sehr eng am Original produzierte Version von Peter Frampton aus dem Jahre 2003, diesem Bild der weinenden Gitarre adäquat Ausdruck zu verleihen. Und möglicherweise kann man in diesem Falle sogar Theodor Gracyk Recht geben, dass diese Aufnahme den Status eines Werks einnimmt, an dem alle weiteren Coverversionen sich zu messen haben (vgl. Gracyk 1996: 37ff.). Besser und im entsprechenden Sinne virtuoser kann man dies wohl nicht gestalten. ${ }^{3}$

Nun kommt es in der Popmusik allerdings nur äußerst selten vor, dass eine Songidee so eng mit einer musikalischen Soundidee verwoben ist wie in »While My Guitar Gently Weeps«. Zumeist liegen bei einem Song ja lediglich ein Text und ein bestimmtes Harmonieschema vor, was zusammen mit der Aufzeichnung des Strophen-Refrain-Verlaufes das Leadsheet bildet, an dem sich Popmusiker zumeist orientieren. Ein solches Leadsheet kann aber nun keineswegs als Werk im Sinne einer ausgearbeiteten musikalischen Struktur bezeichnet werden bzw. würdigt man Popmusik im Vergleich zu komplex ausgearbeiteten strukturorientierten Werken von vornherein ab, wenn man dies als Werk behandelt. Vielmehr lässt sich eher in Anschluss an Daniel Martin Feiges Philosophie des Jazz (vgl. Feige 2014: 83f.) auch von Popmusik als »Musik ohne Werk « sprechen und den Primat bei Popmusik auf die Musikrealisierung legen. Ohne das hier weiter auslegen zu können, stellt sich aber dann doch die Frage, in welcher Weise die oben dargelegten drei Virtuosenbegriffe noch auf Popmusik beziehbar bleiben, wenn kein Werk

a book at random - opened it - saw > Gently weeps - - then laid the book down again and started the song."

3 Lediglich die ironische Brechung dieses Themas scheint hier eine interessante Weiterführung zu sein, wie sie in der Version von Marc Ribot (1990) vorliegt, der im Kontrast zum sanften Wimmern eher ein stoßweises Gitarrenschreien hervorzaubert. 
und entsprechend auch keine feste, in einer Partitur niedergelegte musikalische Idee vorliegt?

Es wurde an anderer Stelle ausführlicher dargelegt (vgl. Stederoth 2017), dass zur ästhetischen Untersuchung einer partiturorientierten Musik Tonalität, Rhythmik/Metrik und Komposition als drei Strukturkategorien zugrunde gelegt werden können, die ja auch oben der Dreiteilung des Virtuosenbegriffs als Orientierung dienten. Diesen drei Kategorien entsprechen dann Felder einer realisierungsorientierten Musik, die sich nicht auf eine Partitur bezieht, die Kategorien Sound, Groove und Performance. Ohne diesen Ansatz hier vertiefen zu können, sei nur so viel gesagt, dass die beiden Bereiche der Struktur- und der Realisierungskategorien sich insofern gegenüberstehen, als die mit ihnen verbundenen Musiken jeweils von einem anderen Primat ausgehen. Während partiturbezogene Musik ihren Primat eben in der musikalischen Struktur und der in ihr verfassten Idee hat und die Realisierung dieser Idee als Interpretation immer an diesem Primat orientiert ist, zeigt sich in der realisierungsorientierten Musik eine Umkehrung des Primats, insofern hier von Sound, Groove und Performance ausgegangen wird und die musikalische Struktur eher sekundären Charakter hat.

$\underline{\text { Ideelle Struktur }}$

musikästhetisches Spanungsfeld

$\underline{\text { Realisierung }}$

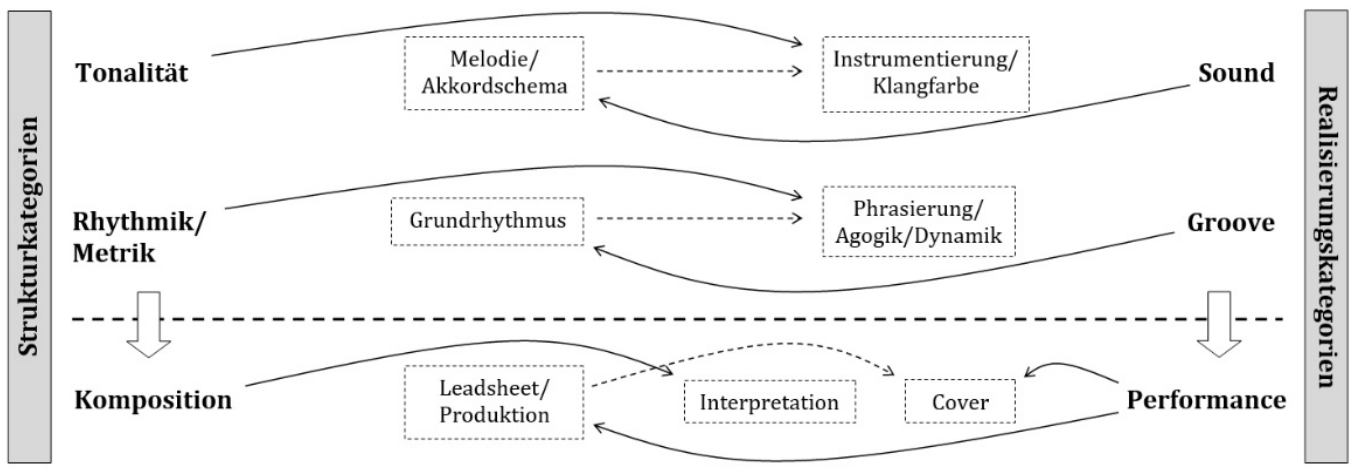

Verstehen

Rezeption

Erleben

Abbildung 1: Musikästhetisches Spannungsfeld

Bezieht man dies nun auf die drei Virtuosenbegriffe, so bedeutet dies, dass dem tonalen Virtuosen, der in seiner Interpretation die musikalische Idee bis in den einzelnen Ton hinein färben muss, der Sound-Virtuose gegenübersteht, der von der Soundgenerierung ausgeht und diese erst sekundär auf eine tonale/harmonische Struktur bezieht. Mag sein, dass Harrison seine Idee aus einem Buch hatte - die Regel ist in der Popmusik, dass beim Expe- 
rimentieren mit Sounds ein interessanter Sound die Inspirationsquelle für eine Songidee ist. So erzählt, wäre die Story gleichermaßen, ja für popmusikalische Kompositionen in aller Regel vielleicht sogar noch plausibler. Entscheidend ist jedoch, dass der Song auch ohne den Hinweis auf die »sanft weinende Gitarre « eine Sound-Virtuosität darstellt. Und vergleicht man die Originalversion mit Harrisons erster Aufnahmesession, die nur mit Akustikgitarren und Orgel aufgenommen wurde, dann wird deutlich, wie viel Sound-Virtuosität in der Originalfassung steckt und vor allem auch, wie sehr Popmusik durch den Sound lebt.

Wenn hier nun von Sound-Virtuosität gesprochen wird, dann sollte man nicht sogleich an die riesigen Effektracks eines John Petrucci denken, insofern Effekte zur Modulierung eines Gitarrensounds lediglich einer von vielen Parametern ist, die zu der Kategorie Sound gehören, wobei der wirkliche Sound-Virtuose die Fähigkeit hat, alle diese Parameter zu einer stimmigen Einheit zusammenzubringen. Wie man an »While My Guitar Gently Weeps« sehr schön hören kann, reichen nur wenige Effekte für einen virtuosen Sound aus, wenn die Lautstärke, die Spielweise, der Anschlag, das Instrument etc. pp. in einer gelungenen Weise sich ergänzen und potenzieren. Viele mit digitalen Effektracks produzierte Sounds verlieren gerade durch die mehrfache digitale Bearbeitung ihre Dynamik im Anschlag, den Eigensound des Instruments und andere wesentliche Parameter, die zur Soundgestaltung beitragen können. Der wirkliche Sound-Virtuose hingegen ist bestrebt, die Ergänzung der unterschiedlichen Parameter möglichst gewinnbringend zu gestalten. Dass dies keineswegs weniger komplex ist als die angemessene farbliche Interpretation einer musikalischen Idee, die den tonalen Virtuosen auszeichnet, wird angesichts der Vielzahl der zu berücksichtigenden Parameter deutlich (siehe Abbildung 2 auf S. 60).

Aber nicht nur ein einzelnes Instrument kann sound-virtuos gespielt sein, sondern es kann ebenso ein gesamter Bandsound virtuos sein, und es versteht sich von selbst, dass hiermit nicht gemeint ist, dass eine Mehrzahl der Bandmitglieder befähigt ist, eine Unzahl an schwierigen Passagen in atemberaubendem Tempo spielen zu können. Ganz im Sinne von Adornos Anmerkung zum gelungenen Kammerorchesterspiel, jedoch übertragen auf die Sphäre des Sounds, lässt sich sagen, dass ein Bandsound dann virtuos gestaltet ist, wenn alle Soundbestandteile zu einer günstigen Einheit sich verbinden. Selbstredend sind für eine solche Virtuosität nicht nur die Bandmitglieder verantwortlich, sondern im Live-Kontext gleichermaßen der Mixer, wie im Studio-Setting zusätzlich noch der Produzent. Dies sei hier jedoch nicht vertieft, sondern vielmehr auf die zweite Realisierungskatego- 


\section{Parameter einer Soundanalyse}

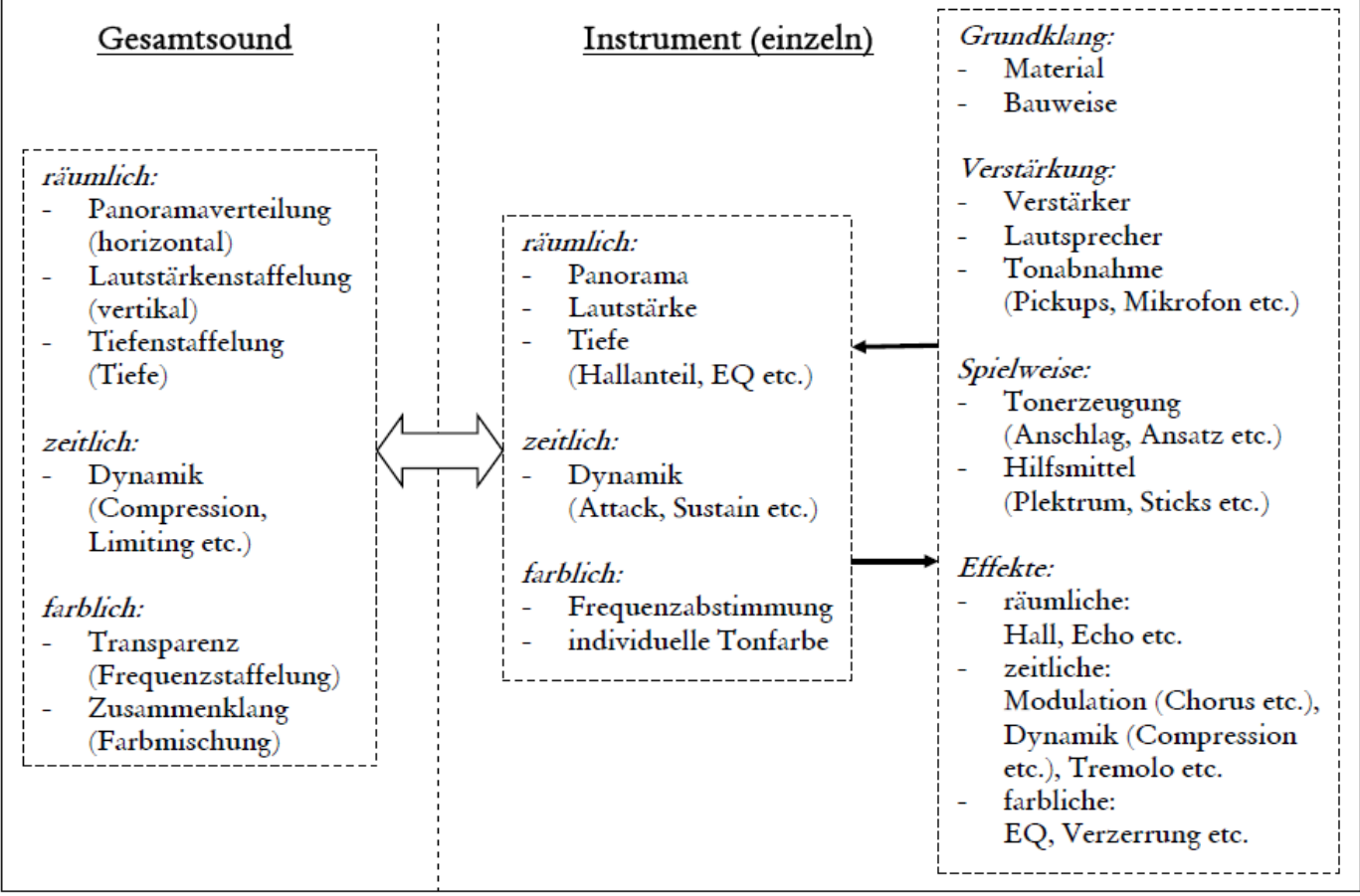

Abbildung 2: Parameter einer Soundanalyse

rie Groove und die Frage eingegangen, was in ihrem Rahmen unter Virtuosität verstanden werden könnte.

Blickt man zunächst auf die jüngeren Forschungen zum Thema »Groove« in der populären Musik die Groove als mikrorhythmische Abweichungen vom Taktmaß fassen (vgl. den Überblick in Pfleiderer 2006: 84ff.), so muss man sagen, dass mit der bloßen Feststellung dieses Tatbestandes für die Klärung der Frage, was denn Groove wirklich sei, recht wenig gewonnen ist. Einen Schritt weiter geht dagegen Charles Keil, wenn er den Begriff der "participatory discrepancies« als Grundlage für Groove einführt (vgl. Keil 1994), da in diesem Begriff die für das Phänomen Groove eigentümliche Spannung mitschwingt. Führt man diesen Gedanken weiter, dann zeichnet Groove gerade die Abweichung vom gegebenen Maß aus, die in dieser Spannung nicht den Bezug zu diesem Maß verliert. Groove kann also nur dann entstehen, wenn die Abweichung des Spiels solchermaßen gestaltet ist, dass das Maß als imaginärer Fokus immer noch mitschwingt bzw. als Fokus permanent »umspielt « wird, wodurch er lediglich indirekt zum Ausdruck gebracht wird.

Die Fähigkeit, ein solches Umspielen möglichst kreativ zu gestalten, ist aber nur eine Komponente, die eine Groove-Virtuosität kennzeichnet. Eine zweite deutet sich in dem an, was man gemeinhin als den »Atem « eines 
Songs oder eines Grooves bezeichnet. Ein Groove braucht »Luft zum Atmen«, weshalb eine permanente Erhöhung der Spielfrequenz von Tönen, die mit dem klassischen Virtuosenbegriff einhergeht, nicht unbedingt förderlich für die Entstehung von Groove ist, ihr manchmal sogar gerade entgegenwirken kann, denn das Umspielen des imaginären Fokus lebt häufig insbesondere durch die geschickte Auslassung von Tönen. Entsprechend kann die kreative und angemessene Wahl von Pausen als eine weitere Komponente für eine Groove-Virtuosität gelten.

Eine dritte Komponente deutet sich an, wenn man sich vergegenwärtigt, dass unterschiedliche Groove-Formen differenziert werden können. Es wurde an anderer Stelle ausführlicher erörtert (vgl. Stederoth 2017), dass sich in einem ersten Schritt vier solcher Formen phänomenologisch unterscheiden lassen: 1. Drive (treibend, Polka), 2. Groove (kreisend, Soul), 3. Swing (wellenförmig, Jazz) und 4. Boogie (spiralig, Disco). Es liegt auf der Hand, dass jede dieser Groove-Formen eine eigenständige Art des Umspielens erfordert, weshalb der Groove-Virtuose auch die jeweils angemessene Form des Umspielens zum Ausdruck bringen muss. Dabei wäre zu untersuchen bzw. gilt es als offene Frage, ob wirkliche Virtuosität nicht immer nur in einer dieser Formen erreicht werden kann oder ob sich Musiker auch in unterschiedlichen Groove-Formen virtuos ausdrücken können. Dies muss aber zunächst ebenso als ungeklärt gelten wie die Frage nach einer weiteren Differenzierung solcher Grundformen des Grooves.

Abschließend sei noch darauf hingewiesen, dass jenes Umspielen zwar auch in einem solistischen Spiel (ohne weitere Musiker) möglich ist, sich in der populären Musik jedoch zuallermeist in einem Bandkontext vollzieht. Dabei kommt es (wie schon bei der Erörterung der Sound-Virtuosität) darauf an, dass die unterschiedlichen Glieder einer Band gemeinsam ein solches Umspielen bewerkstelligen. Das bedeutet für eine groove-virtuose Band, dass alle Bandmitglieder sich in den verschiedenen Komponenten produktiv ergänzen, damit, um ein Beispiel zu geben, eine kreativ gesetzte Pause des Bassisten nicht durch ein massives Spiel eines Gitarristen wieder überspielt wird. Jedoch führen diese Erörterungen bereits zur dritten Realisierungskategorie, die mit »Performance sich insbesondere auf das Zusammenspiel der Bandmitglieder richtet, was nun in den Fokus treten soll.

Es wurde am Beginn dieses Abschnitts schon im Rekurs auf eine Aussage von Satriani darauf hingewiesen, dass sich die Grundtugenden des Zusammenspiels in einer Rockband nur wenig von denen eines Kammerorchesters, wie sie von Adorno beschrieben wurden, unterscheiden. Es sei jedoch an dieser Stelle auch auf einen Unterschied verwiesen, der zentral für das sein könnte, was eine Performance-Virtuosität auszeichnet. Der Unterschied zwi- 
schen einer strukturorientierten Interpretation und einer realisierungsorientierten Performance liegt zunächst darin, dass - wie oben erörtert - eine Interpretation sich an einer vorgegebenen Struktur, dem Werk orientiert, während das für eine realisierungsorientierte Performance nicht in gleicher Weise gelten kann. In seiner Philosophie des Jazz hat Feige (2014) herausgearbeitet, dass es sich bei Standard-Jazz vielmehr um eine Musik handelt, die fast vollständig aus dem Aktual der musikalischen Performance heraus verstanden werden muss, insofern Tonales und Rhythmisches fast vollständig während der Performance verhandel- und veränderbar sind. Bezieht man diesen Ansatz nun auf Popmusik im engeren Sinne, so lässt sich sagen, dass die Verhandel- und Veränderbarkeit fast gleichermaßen für sie zutrifft, allerdings mit dem Unterschied, dass die Verhandlungen bereits vor der Performance getroffen werden, obgleich es zu einer guten Pop-, Rock- oder Soul-Performance ebenso gehört, dass die Musiker während des Spielens aufeinander reagieren und spontane Variationen kreieren, was aber bei Weitem nicht in dem Umfang geschehen kann, wie das im Standard-Jazz der Fall ist. Demgegenüber kommt der Performance im Popbereich noch eine Komponente zu, die für den Jazz weniger zentral ist und das ist die Einbeziehung des Publikums in die Performance. Dies kann in der Popmusik soweit gehen, dass das Publikum gleichsam zu einem weiteren Bandmitglied wird, insofern die Kommunikation zwischen Band und Publikum die gesamte Performance trägt. Auch wenn eine solche Publikumsorientierung gleichermaßen virtuos gestaltet sein kann (man denke etwa an Mick Jagger), soll sie hier nicht weiter untersucht werden.

Vielmehr seien nochmal die mehr musikalischen Merkmale einer Performance-Virtuosität genauer in den Blick genommen. Neben den bereits an entsprechender Stelle angedeuteten Sound- und Groove-Komponenten sei noch auf eine weitere Komponente hingewiesen, die jene beiden ergänzt, jedoch zugleich auch eine Basis für diese darstellt: Es ist das kreative Wechselspiel von spontanen Einfällen und Variationen. Virtuos ist eine StandardJazzband dann, wenn alle beteiligten Musiker idealerweise permanent offen sind für die Einfälle und Variationen der jeweils anderen Musiker und zugleich auf der Basis dieser Einfälle und Variationen eigene spontane Einfälle und Variationen in das Wechselspiel einbringen. Da dieser ideale Fall eine Gleichzeitigkeit von wacher Aufmerksamkeit und kreativer Produktion erfordern würde, die letztlich in absoluter Weise nicht erreichbar ist, deutet diese Gleichzeitigkeit doch eine Zielrichtung an, auf die hin eine Performance-Virtuosität sich bewegt. Zugleich kommt in diesem Wechselspiel, und zwar insbesondere in der Popmusik, dem Verhältnis von variativen und stabilisierenden Elementen eine besondere Bedeutung zu, wobei alle Band- 
mitglieder gleichzeitig ihr Augenmerk darauf richten müssen. Stört dieser Einfall den Gesamtgroove? Verändert dieses Lick die aktuale Spannungsentwicklung des Songs? Stört die variative Änderung der Lage, in der ein Lauf gespielt wird, den Gesamtsound? Solche und Unmengen ähnlicher Fragen müssen während einer Performance populärer Musik in actu entschieden und angemessen umgesetzt werden. Die Komplexität dieses Geschehens steigert sich natürlich immens dadurch, dass jedes Bandmitglied parallel mit solchen Fragen und Entscheidungen konfrontiert ist, wobei die jeweiligen Entscheidungen unmittelbar wiederum in die jeweils eigenen weiteren Erwägungen einfließen müssen. Dieses äußerst komplexe Geschehen macht überdeutlich, dass Performance ein weites Feld für die Ausbildung von virtuosen Fähigkeiten bildet, die erst einmal wenig mit technischer Brillanz zu tun haben.

Stellte sich eingangs die Frage, ob der klassische Virtuosenbegriff, der insbesondere mit technischen Fähigkeiten im Zusammenhang steht, nicht durch weitere Virtuosenbegriffe ergänzt werden kann, so wurde im Verlauf des Textes versucht zu zeigen, dass mindestens sechs solcher Formen sich bestimmen lassen, wobei drei mehr in das Feld strukturorientierter Musik fallen (tonale, rhythmische und Ensemble-Virtuosität) und drei weitere sich aus dem Feld einer realisierungsorientierten Musik herleiten (Sound-, Groove- und Performance-Virtuosität). Der Vorteil einer solchen Differenzierung von Virtuosität ist nicht nur, dass in Bezug auf ihn dezidiert musikalischen Kategorien in den Blick treten, sondern auch, dass es durch sie zuallererst möglich wird, die eher stillen Virtuosen in der Musik ausfindig zu machen, so dass das Spiel eines George Harrison möglicherweise als weit virtuoser bezeichnet werden kann als die Griffbretteskapaden mancher Gitarren-Sportler, denen eine »sanft weinende Gitarre« nur schwerlich aus den Fingern rinnt.

\section{Literatur}

Adorno, Theodor W. (1975). Einleitung in die Musiksoziologie. Zwölf theoretische Vorlesungen. Frankfurt/M.: Suhrkamp.

Adorno, Theodor W. (2014). Kranichsteiner Vorlesungen. Hg. v. Klaus Reichert und Michael Schwarz. Berlin: Suhrkamp.

Feige, Daniel Martin (2014). Philosophie des Jazz. Berlin: Suhrkamp.

Gracyk, Theodore (1996). Rhythm and Noise. An Aestethics of Rock. London, New York: I.B. Tauris.

Harrison, George (1980). I, Me, Mine. Guildford: Genesis Press.

Keil, Charles / Feld, Steven (1994). Music Grooves. Chicago: University of Chicago Press. 
Menge, Marian (2016). »G3 2016. Joe Satriani \& Steve Vai über eine Institution. «In: Gitarre \& Bass, H. 9 (September), S. 32-41.

Pfleiderer, Martin (2006). Rhythmus. Psychologische, theoretische und stilanalytische Aspekte populärer Musik. Bielefeld: transcript.

Reimer, Erich (1973). »Die Polemik gegen das Virtuosenkonzert im 18. Jahrhundert. Zur Vorgeschichte einer Gattung der Trivialmusik.« In: Archiv für Musikwissenschaft 30, H. 4, S. 235-244.

Riemann, Hugo (1884). Musikalische Dynamik und Agogik. Lehrbuch der musikalischen Phrasierung auf Grund einer Revision der Lehre von der musikalischen Metrik und Rhythmik. Hamburg, Leipzig, St. Petersburg: Rahter, Kistner, Büttner.

Riemann, Hugo (1967). »Das Überhandnehmen des musikalischen Virtuosentums.« In: Ders., Präludien und Studien I. Hildesheim: Georg Olms, S. 3-12.

Stederoth, Dirk (2017). "Sound, Groove, Performance. Musikästhetische Realisierungskategorien zur Charakterisierung populärer Musik.« In: Was ist Popmusik? Konzepte, Kategorien, Kulturen. Hg. v. Timo Hoyer, Carsten Kries und Dirk Stederoth. Darmstadt: Wissenschaftliche Buchgesellschaft (im Druck).

Zappa, Frank / Occhiogrosso, Peter (1989). The Real Frank Zappa Book. New York u.a.: Poseidon Press.

\title{
Diskografie
}

Frampton, Peter (2003) »While My Guitar Gently Weeps. Auf: Now. CD. 33rd Street Records 3321.

Ribot, Marc (1990). »While My Guitar Gently Weeps. " Auf: Rootless Cosmopolitans. CD. Antilles 314-510 091-2.

The Beatles (1968). „While My Guitar Gently Weeps." Auf: The Beatles [White Album]. 2 LPs. Apple Records PCS 7067/8.

Zappa, Frank (1986). Jazz From Hell. LP: Barking Pumpkin Records ST 74205.

\begin{abstract}
Based on the assessment that the classical notion of the virtuoso as defined by his or her technical prowess is also prevalent within the sphere of popular music, this text initially engages with the classic critiques of this term. Following a musicaesthetic differentiation of structure-oriented and realization-oriented music, the text first develops three notions of the virtuoso for notation-based, structureoriented music (tonal, rhythmic, and ensemble virtuosity) in order to distill corresponding definitions of the virtuoso within the sphere of realization-oriented music (sound, groove and performance virtuosity) that prove themselves to be pertinent to a clearer determination of virtuosity in popular music, which is substantiated on the example of the song »While My Guitar Gently Weeps«.
\end{abstract}

